\title{
Introduction to the focused issue on the 20th International Conference on Theory and Practice of Digital Libraries (TPDL 2016)
}

\author{
Norbert Fuhr ${ }^{1}$ • László Kovács ${ }^{2} \cdot$ Thomas Risse $^{3} \cdot$ Wolfgang Nejdl $^{4}$
}

Published online: 1 February 2019

๑) Springer-Verlag GmbH Germany, part of Springer Nature 2019

Valuable and rapidly increasing volumes of data are created or transformed into digital form by all fields of scientific, educational, cultural and governmental and industry activities. For this purpose, the digital libraries community has developed long-term and interdisciplinary research agendas, providing significant results, such as development of digital libraries, solving practical problems, accommodating research data and satisfying the needs of specific user communities.

The advent of the technologies that enhance the exchange of information with rich semantics is of particular interest in the community. Information providers interlink their metadata with user-contributed data and offer new services out looking for the development of a web of data and addressing the interoperability and long-term preservation challenges.

The 20th edition of the International Conference on Theory and Practice of Digital Libraries (TPDL) held in Hannover, Germany, during September 5-9, 2016, had the general theme of "Overcoming the limits of Digital Archives" and focused on the major areas: "Connecting digital libraries," "Practice of digital libraries," "Digital libraries in science" and "Users, communities, and personal data." In addition, specialized track on "Digital Humanities," "eInfrastructures" and "Creativity and Multimedia Libraries" were presented.

All submissions to the conference were independently reviewed on the basis of peer review process. A Senior Program Committee member subsequently coordinated a discussion among the reviewers. The selection stage that followed compared the paper evaluations and finalized the conference program. Eight papers with the highest score and

\footnotetext{
Norbert Fuhr nejdl@13s.de

1 Universität Duisburg-Essen, Duisburg, Germany

2 Hungarian Academy of Sciences, Budapest, Hungary

3 University Library Johann Christian Senckenberg, Frankfurt, Germany

4 Leibniz Universität Hannover, Hannover, Germany
}

the best paper of each specialized track were selected as candidates for the Special Issue of the International Journal on Digital Libraries, asking the authors to introduce at least $30 \%$ difference from the conference original paper. The new submissions were reviewed by four reviewers, common with the original TPDL 2016 reviewers if possible.

The earlier version of the paper in "Discovering the structure and impact of the digital library evaluation domain" by Leonidas Papachristopoulos, Giannis Tsakonas, Moses Boudourides, Michalis Sfakakis, Nikos Kleidis, Sergios Lenis and Christos Papatheodorou received the best paper award of TPDL 2016. The authors propose a methodological pathway to investigate the core topics that structure the digital library evaluation domain and their impact. The multidimensional nature of digital libraries evaluation domain and the amount of scientific production that is published in the domain hinders and disorientates the interested researchers. The proposed methodology aims at guiding researchers to exploit effectively the considerable amount of data and the diversity of methods, as well as to identify new research goals. It exploits topic modeling and network analysis in combination with citation and altmetrics analysis on a corpus consisting of the digital library evaluation papers presented in JCDL, ECDL/TDPL and ICADL conferences in the period 2001-2013.

In "Benchmarking and evaluating the interpretation of bibliographic records" by Trond Aalberg, Fabien Duchateau, Naimdjon Takhirov, Joffrey Decourselle and Nicolas Lumineau, a first benchmark for the FRBRization of bibliographic records is presented. The Functional Requirements for Bibliographic Records (FRBR) and its updated version Library Reference Model (LRM) are designed to provide explicit semantics for bibliographic metadata. To evaluate the quality of tools that migrate legacy data to FRBR, the paper proposes a benchmark for the quality assessment. The main contribution of the work is the definition of new metrics that estimate the quality and the efficiency of the migration process and to evaluate the quality of a FRBRized catalogue. For the application of the benchmark, two public datasets 
have been released. The paper analyses the applicability of the benchmark and presents its limitations.

The retrieval of videos content based on concepts, persons, textual descriptions or similarity search is the topic of the paper by Markus Mühling, Manja Meister, Nikolaus Korfhage, Jörg Wehling, Angelika Hörth, Ralph Ewerth and Bernd Freisleben. In "Content-based video retrieval in historical collections of the German Broadcasting Archive," the authors present their approach for the concept classification based on deep convolutional neural networks. They present in addition a novel, fast similarity search for large-scale video retrieval. The performance of the system has successfully been evaluated on $2500 \mathrm{~h}$ of television material from the former German Democratic Republic (GDR).
In the work by João Rocha da Silva, Cristina Ribeiro and João Correia Lopes on "Ranking Dublin Core descriptor lists from user interactions: a case study with Dublin Core Terms using the Dendro platform" the authors aim to support the curation of Dublin Core Application Profiles. The approaches use past user behavior to discover and use adequate descriptors. The approach has been developed in the context of research data management to ease and motivate users to described research data sets.

Publisher's Note Springer Nature remains neutral with regard to jurisdictional claims in published maps and institutional affiliations. 